\title{
Alimentação precoce no pós-operatório de cirurgias digestivas
}

\author{
Early feeding in the postoperative period of digestive surgery \\ Alimentación temprana en el postoperatorio de cirugía digestiva \\ Lucas Santana Soares ${ }^{1 \star}$, Marcelo Protásio dos Santos ${ }^{1}$, Erika de Oliveira Menezes ${ }^{1}$.
}

\begin{abstract}
RESUMO
Objetivo: Avaliar os benefícios da alimentação precoce no pós-operatório de cirurgias digestivas. Métodos: Trata-se de uma revisão integrativa de literatura cujo tema foi pesquisado na base de dados da Scielo e Pubmed através dos descritores "alimentação no pós-operatório" e "postoperative feeding", respectivamente, sendo incluídos artigos com pacientes maiores de 18 anos e sem restrição à data de publicação. Resultados: A revisão dos artigos selecionados evidenciou que o manejo tradicional da alimentação pós-operatória está ultrapassado e a formulação dos novos protocolos como Enhanced Recovery After Surgery (ERAS) e Aceleração da Recuperação Total Pós-Operatória (ACERTO) possibilitam uma mudança benéfica nessa conduta. A alimentação precoce no pós- operatório é responsável por acelerar a recuperação do paciente, bem como, por reduzir os custos hospitalares durante a internação do paciente. Esses benefícios são constatados tanto em cirurgias abertas quanto em videocirurgias. Outro aspecto importante é a região anatômica do procedimento, sendo as cirurgias colorretais as mais estudadas e com resultados positivos mais consistentes. Considerações Finais: A alimentação precoce no pós-operatório de cirurgias colorretais e cirurgias digestivas altas é segura, reduzindo a morbimortalidade e o tempo de internação hospitalar.
\end{abstract}

Palavras-chave: Alimentação, Cuidados pós-operatórios, Cirurgia.

\begin{abstract}
Objective: Evaluate the benefits of early feeding in the postoperative period of digestive surgery. Methods: It is an integrative literature review whose theme was researched in the Scielo and Pubmed database through the descriptors "alimentação no pós-operatório" and "postoperative feeding", respectively, including articles with patients over 18 years old and without restriction the date of publication. Results: The review of the selected articles showed that the traditional management of postoperative feeding is outdated and the formulation of new protocols such as Enhanced Recovery After Surgery (ERAS) and Aceleração da Recuperação Total Pós-Operatória (ACERTO) allows for a beneficial change in this conduct. Early feeding in the postoperative period is responsible for accelerating recovery in the postoperative period as well as reducing hospital costs during the patient's hospitalization. These benefits are seen in both open and video surgeries. Another important aspect is the anatomical region of the procedure, with colorectal surgeries being the most studied and with the most consistent positive results. Final Considerations: Early feeding in the postoperative period of colorectal surgery and upper digestive surgery is safe, reducing morbidity, mortality and hospital stay.
\end{abstract}

Key words: Feed, Postoperative care, Surgery.

\section{RESUMEN}

Objetivo: Evaluar los beneficios de la alimentación temprana en el postoperatorio de cirugía digestiva. Métodos: Se trata de una revisión integradora de la literatura cuyo tema fue investigado en la base de datos Scielo y Pubmed a través de los descriptores "alimentação no pós-operatório" y "alimentación postoperatoria", respectivamente, incluyendo artículos con pacientes mayores de 18 años y sin restricción. la fecha de

${ }^{1}$ Universidade Tiradentes (UNIT), Aracaju - SE. *E-mail: lucas.soaress94@gmail.com 
publicación. Resultados: La revisión de los artículos seleccionados mostró que el manejo tradicional de la alimentación postoperatoria está desactualizado y la formulación de nuevos protocolos como Enhanced Recovery After Surgery (ERAS) e Aceleração da Recuperação Total Pós-Operatória (ACERTO) permite un cambio beneficioso en esta conducta. La alimentación temprana en el postoperatorio es responsable de acelerar la recuperación en el postoperatorio así como de reducir los costos hospitalarios durante la hospitalización del paciente. Estos beneficios se ven tanto en cirugías abiertas como en video. Otro aspecto importante es la región anatómica del procedimiento, siendo la cirugía colorrectal la más estudiada y con resultados positivos más consistentes. Consideraciones finales: La alimentación temprana en el postoperatorio de cirugías colorrectales y cirugías digestivas altas es segura, reduciendo la morbilidad y mortalidad y la estancia hospitalaria.

Palabras clave: Alimentación, Cuidados posoperatorios, Cirugía.

\section{INTRODUÇÃO}

No decorrer dos anos houve importante desenvolvimento tecnológico nos campos da cirurgia e da anestesia. Diversas condutas perioperatórias, comumente adotadas e repassadas empiricamente ao longo de anos, hoje mostram-se ultrapassadas à luz das evidências, devendo ser, portanto, abandonadas (AGUILAR-NASCIMENTO JE, et al., 2017).

De acordo com Le Guen M, et al. (2014), a abordagem tradicional ao atendimento pós-operatório de cirurgia abdominal retarda a nutrição até a liberação de flatos ou movimento intestinal. Essa prática compromete o estado nutricional desses pacientes e, consequentemente, pode prejudicar a evolução do pósoperatório com uma importante e conhecida resposta ao estresse catabólico. Tal prática faz com que o jejum pós-cirúrgico se prolongue por até cinco dias em média. Além disso, a tradição cirúrgica orienta a oferta escalonada ou progressiva da dieta líquida, pastosa até à sólida, o que pode contribuir com a piora do estado nutricional de pacientes previamente desnutridos e aumento do tempo de internação hospitalar (CORREIA MITD e SILVA RG, 2005; FRANCISCO SC, et al., 2015; DI FRONZO LA, et al., 2003).

Novas evidências têm demonstrado que o abreviamento do jejum é capaz de promover um retorno mais rápido da função gastrointestinal, favorecer a recuperação metabólica, reduzir o tempo de internação hospitalar e diminuir taxas de complicações pós-operatórias e custos hospitalares (FRANCISCO SC, et al., 2015). Sendo assim, a nutrição adequada para o paciente cirúrgico é fundamental para boa evolução clínica e redução de complicações pós-operatórias (AGUILAR-NASCIMENTO JE, et al., 2007).

Com o objetivo de diminuir as complicações cirúrgicas e acelerar a recuperação dos pacientes, um grupo de pesquisadores desenvolveu, em 2001, um protocolo de recuperação pós-operatória, Enhanced Recovery After Surgery (ERAS), apresentando várias alterações nas formas tradicionalistas de cuidados, baseadas em estudos controlados, randomizados e meta-análises (AGUILAR-NASCIMENTO JE, et al., 2006; SÁNCHEZ A e PAPAPIETRO K., 2017).

Para adaptar as mudanças propostas pelo projeto ERAS à realidade nacional, em 2005, o Departamento de Clínica Cirúrgica da Faculdade de Ciência Médicas da Universidade Federal do Mato Grosso desenvolveu o projeto Aceleração da Recuperação Total Pós-Operatória (ACERTO). A Medicina Baseada em Evidências tem demonstrado que protocolos de aceleração da recuperação pós-operatória, baseados no ACERTO, são seguros, reduzem complicações pós-operatórias e o tempo de internação, sem acréscimo nas taxas de reinternação. Esses protocolos incluem diversos fatores, entre eles a reintrodução da dieta no primeiro dia de pós-operatório (6-24 horas) para a maioria das cirurgias (FRANCISCO SC, et al., 2015).

Desse modo, a preocupação nutricional com os pacientes cirúrgicos é um dos pilares do cuidado perioperatório, que pode ser ajustado para melhorar o desfecho clínico dos doentes, seguindo as recomendações de protocolos como o ERAS e ACERTO. Nesse sentido, propõe-se um estudo para avaliar os benefícios da alimentação precoce no pós-operatório de cirurgias digestivas. 


\section{MÉTODOS}

Trata-se de uma revisão de literatura realizada por meio da coleta de informações através de levantamento bibliográfico seguindo os moldes de uma revisão integrativa. Desse modo, busca analisar uma dada temática, já abordada anteriormente, com o objetivo de melhor compreendê-la. A pesquisa foi desenvolvida a partir dos seguintes passos: identificação do tema e seleção da questão de pesquisa; estabelecimento de critérios de inclusão e exclusão; identificação e seleção dos estudos; categorização dos estudos selecionados; análise e interpretação dos resultados e apresentação da revisão/síntese do conhecimento.

A pesquisa foi realizada nas bases de dados National Library of Medicine (PUBMED) e Scientific Eletrônic Library Online (SCIELO). Foram utilizados os descritores "postoperative feeding" e "alimentação no pósoperatório", respectivamente. Incluíram-se artigos cujo tema principal abordasse a abreviação do jejum pósoperatório em cirurgias gastrointestinais altas e baixas, independentemente da data de publicação. Excluíramse aqueles em que o procedimento cirúrgico não estivesse relacionado ao trato gastrointestinal ou fizessem análise de pacientes menores de 18 anos.

Inicialmente, a busca de artigos que se adequassem aos critérios de inclusão se deu no PUBMED. A pesquisa avançada restringiu-se aos artigos que apresentassem os descritores no título e resumo. Foram encontrados 297 artigos, dos quais apenas 32 apresentavam como tema essencial a abreviação do jejum pós-operatório em cirurgias gastrointestinais altas e baixas. Destes, ainda foram excluídos onze, por abordar pacientes menores de 18 anos ou por estarem relacionados a procedimentos cirúrgicos não relacionados ao trato gastrointestinal. Restaram, então, 21 artigos adequados ao estudo.

Em sequência, foi realizada busca no SCIELO por meio dos filtros: idiomas português e inglês, independentemente do tempo de publicação. Como resultados, foram obtidos 44 artigos, dos quais apenas 13 se adequaram aos critérios de inclusão. Vale salientar, então, que foram selecionados um total de 34 artigos para o presente estudo (Figura 1).

Durante a elaboração da pesquisa não houve necessidade de aprovação do Comitê de Ética em Pesquisa (CEP), uma vez que, por se tratar de uma revisão bibliográfica, somente foram utilizados dados disponibilizados eletronicamente.

Figura 1 - Resultado da pesquisa proposta na metodologia.

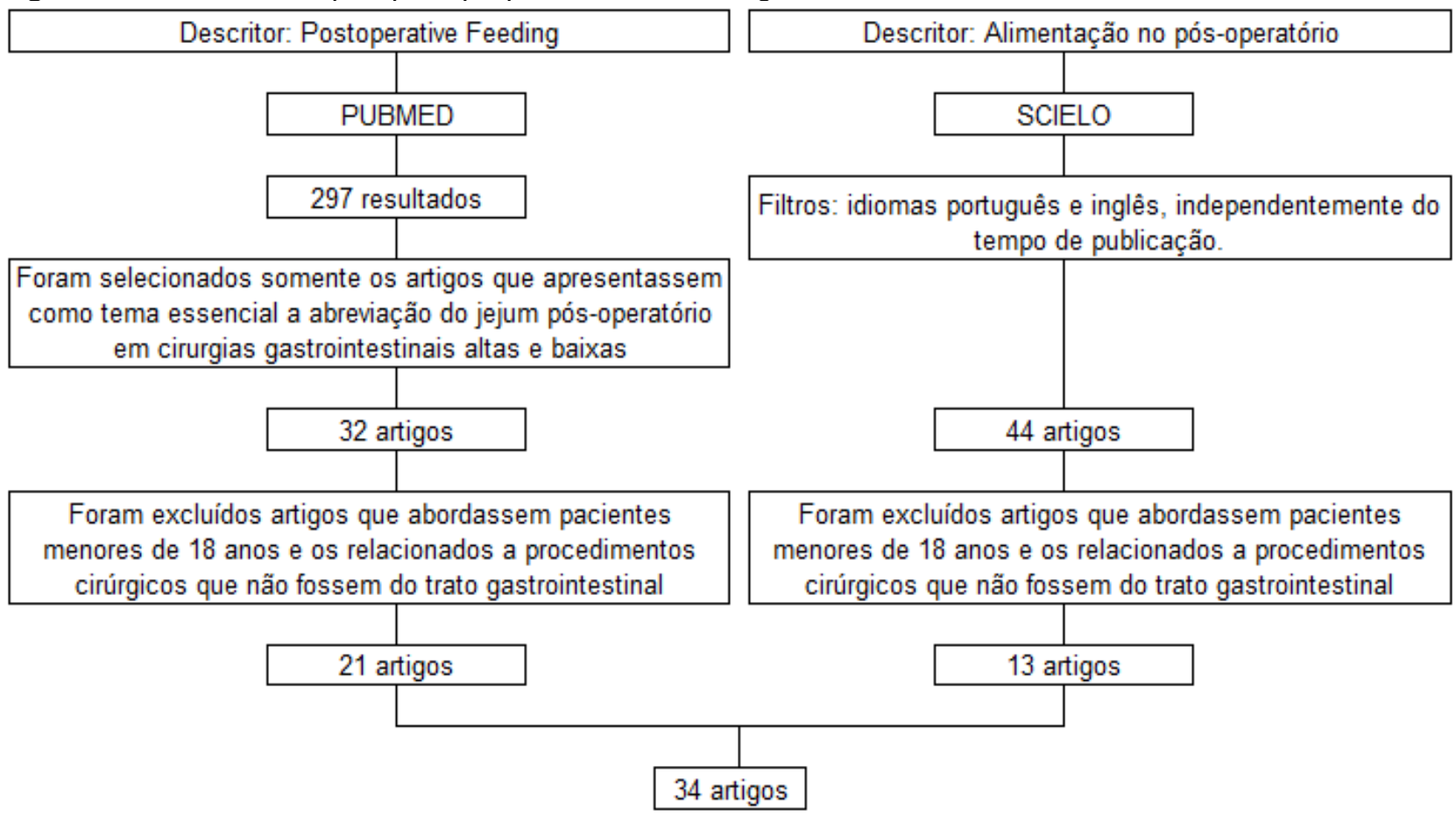

Fonte: SOARES LS, et al., 2020. 


\section{DISCUSSÃO}

Habitualmente, a ingestão oral após a cirurgia é postergada até que a peristalse seja recuperada, geralmente em três a cinco dias. Alguns sinais físicos, incluindo sons intestinais, flatos e defecação, são usados para anunciar o retorno da função intestinal (BUFO AJ, et al., 1994). Existe a crença de que a alimentação precoce no pós-cirúrgico agrava o íleo adinâmico, por ocasionar náuseas, vômitos e distensão abdominal. Entretanto, a ocorrência de tais sintomas está mais vinculada à aplicabilidade de técnicas anestésicas ultrapassadas, do que à abreviação da reintrodução da dieta no pós-operatório. Ainda assim, muitas condutas se baseiam na necessidade de aguardar a resolução do íleo pós-operatório (SILVA VB, et al., 2015).

Segundo Costa HC, et al. (2013) o cuidado pós-operatório tradicional tem sido questionado e novos paradigmas de Medicina baseada em evidência vêm mostrando que, nos últimos anos, algumas rotinas e protocolos no cuidado perioperatório são inúteis e, em alguns casos, prejudiciais. No entanto, alguns aspectos ainda continuam a ser alvo de inúmeras controvérsias, especialmente no tocante ao período adequado de jejum pré-operatório e à liberação da dieta. Esses aspectos envolvem alguns dos paradigmas existentes em Medicina, mais propriamente em cirurgia, que geram, angústia e medo aos profissionais envolvidos no tratamento de pacientes operados (CORREIA MI e SILVA RG, 2005).

Para reforçar a necessidade de modificação da abordagem sobre a dieta pós-operatória em pacientes submetidos a cirurgia abdominal eletiva, Osland E, et al. (2011) realizaram uma metanálise com 15 estudos sendo a primeira a demonstrar reduções estatisticamente significativas nas complicações totais no pósoperatório de cirurgias abdominais com a implementação da alimentação precoce, não evidenciando efeitos negativos com relação à mortalidade hospitalar, deiscência anastomótica, tempo de permanência e recuperação da função intestinal.

Um dos aspectos que amedronta os médicos e os impede de iniciar a dieta após a cirurgia precocemente é o tempo necessário para retorno da função intestinal. Para Gianotti L, et al. (2011) a recuperação da função intestinal após operações abdominais é multifatorial. Entre os fatores mais importantes estão a atividade do sistema nervoso autônomo, a liberação de hormônios gastrointestinais, mediadores inflamatórios e numerosas vias metabólicas teciduais. Além disso, o tipo de anestesia, os medicamentos utilizados para o controle da dor e a gravidade do trauma cirúrgico alteram a ação de um ou mais desses modificadores e, portanto, também podem interferir na motilidade intestinal.

De acordo com Ludwig RB, et al. (2013), no primeiro dia do pós-operatório iniciam-se o esvaziamento do conteúdo gástrico e pequenas absorções intestinais. As atividades do cólon retornam somente 48 horas após a operação. O estômago e o pâncreas secretam um a dois litros de líquidos por dia que são absorvidos no intestino delgado. Sendo assim, pode-se afirmar que mesmo não havendo completo funcionamento do trato gastrointestinal, poderá haver ainda alguma absorção.

Reforçando a dificuldade em iniciar a dieta precocemente, Rattray M, et al. (2018) realizaram uma revisão sistemática com 29 estudos em pacientes submetidos a cirurgias abdominais na qual observou que apenas $40 \%$ e $22 \%$ relataram tempo para a primeira alimentação e tempo para a primeira alimentação sólida em concordância com a recomendação baseada em evidências, respectivamente. Para facilitar a padronização da abordagem à alimentação é preciso uma comunicação multidisciplinar adequada, bem como o reconhecimento dos recursos dietéticos disponíveis no hospital, pois esses são considerados fatores-chave que influenciam no desfecho da introdução da alimentação pós-operatória precoce adequada e oportuna (RATTRAY M, et al., 2020).

Outra questão no cuidado após cirurgias diz respeito ao modo de introdução da dieta. A literatura demonstra que a tradição cirúrgica orienta a oferta escalonada ou progressiva da dieta líquida, pastosa até à sólida. Todavia, essa prática não só pode contribuir com a piora do estado nutricional de pacientes previamente desnutridos, como aumenta o tempo de internação hospitalar (CORREIA MI e SILVA RG, 2005).

A necessidade de se conhecer melhor os prejuízos acarretados pelo jejum pós-cirúrgico estão baseados nos conhecimentos acerca das modificações nutricionais e metabólicas que ocorrem nesses pacientes. Se a 
extensão e o risco da cirurgia não são adaptados à capacidade do paciente de gerar uma resposta adequada ao trauma cirúrgico, há um alto risco de vazamento através da anastomose, complicações infecciosas, podendo evoluir para sepse e mortalidade, essa resposta está diretamente relacionada ao status nutricional do paciente (WEIMANN A, et al., 2017). Portanto, é importante salientar que a desnutrição está associada à perda de tecido e à função orgânica prejudicada, o que leva a aumento da morbidade e a períodos mais longos de hospitalização (DERVENIS C, et al., 2003).

Silva VB, et al. (2015) e Stocche RM, et al. (2001) afirmam que o trauma cirúrgico acarreta uma elevação do gasto energético para favorecer o reparo das lesões, nesse processo as proteínas tornam-se substrato energético para tecidos glicolíticos, dessa forma, ocorre aumento da gliconeogênese e proteólise, isso associado ao jejum prolongado resulta em desnutrição pós-traumática grave.

Outro aspecto relevante é o entendimento de que a resposta orgânica ao estresse é um fenômeno fisiológico, onde múltiplos estímulos atingem o hipotálamo e estimulam o sistema nervoso simpático e a medula supra-renal a liberarem substâncias desencadeadoras da resposta, com intuito de manter a homeostase corporal. Estes estímulos prolongados e de grande intensidade, tornam a resposta ao estresse orgânico exacerbada. Ademais, a produção e liberação de citocinas inflamatórias, desencadeados pela lesão tecidual, acarretam modificações metabólicas importantes e parecem estar associados ao aumento da resistência periférica à insulina (LUDWIG RB, et al., 2013; DE LUIS DA, et al. 2014).

Essa resistência insulínica aumentada associada ao estado de hiperglicemia ocasiona elevação no gasto de oxigênio, bem como retenção de sódio e água, além de favorecer o estado de hipercoagulabilidade, elevando o risco para infarto agudo do miocárdio e eventos tromboembólicos. Ademais, a imunossupressão devido ao cortisol aumentado, e o estado de catabolização instaurado no paciente prejudicam o processo de cicatrização (LUDWIG RB, et al., 2013; SILVA VB, et al., 2015; WEIMANN A, et al., 2017).

Outra questão abordada diz respeito à utilização de sonda nasogástrica como um critério de tolerância a dieta via oral, uma vez que antes da criação dos protocolos de aceleração de recuperação pós operatória uma prática muito comum era a realização desse procedimento em pacientes submetidos a cirurgias abdominais, mas essa necessidade vem sendo questionada com o passar do tempo. A doutrina cirúrgica sustentou que a sondagem nasogástrica é um componente necessário dos cuidados pós-operatórios em cirurgias do aparelho digestivo com base em conhecimentos passados de que a motilidade intestinal é normalmente atrasada e retorna de maneira previsível. Entretanto, estudos comparativos demonstram que a descompressão nasogástrica de rotina é desnecessária, dado que pacientes sondados e pacientes sem sonda apresentam resultados iguais. (ORTIZ H, et al.,1996; HARTSELL P, et al., 1997; AKBARSHAHI H, et al., 2008)

Além disso, também importante é a razão pela qual ocorre intolerância a dieta oral precoce no póscirúrgico. Difronzo LA, et al. (1999) observaram que dentre os fatores de risco associados a essa falha na dieta estavam o sexo masculino, a colectomia total e a proctocolectomia total, mas não demonstraram de maneira objetiva os reais motivos de homens terem maior predisposição a falha. Em contrapartida, o estudo de Petrelli NJ, et al. (2002) não demonstrou interferência do sexo na alimentação oral precoce. De modo que esse fato estaria melhor relacionado ao tipo de cirurgia realizada, como colectomia total. Petrelli $\mathrm{NJ}$, et al. (2002) e Dervenis C, et al. (2003) demonstraram que os pacientes que falharam durante a realimentação pósoperatória precoce receberam mais expansores de volume e apresentaram maior perda sanguínea se comparado aos demais pacientes, sugerindo que essa prática contribui para o edema da parede intestinal e íleo prolongado.

Outro aspecto importante diz respeito ao tipo de cirurgia, seja ela aberta ou laparoscópica. Na abordagem tradicional acreditava-se que a alimentação precoce somente era possível em pacientes submetidos a cirurgias laparoscópicas, por apresentarem menor trauma cirúrgico, entretanto esse paradigma já foi questionado inclusive por estudos mais antigos. Segundo Binderow SR, et al. (1994) pacientes submetidos a laparotomia, assim como aqueles que tiveram um procedimento laparoscópico são geralmente capazes de tolerar a ingestão oral precoce. 
A alimentação precoce e seus desfechos sofrem influência direta da região anatômica do procedimento realizado no paciente. O estudo de Atkinson C, et al. (2020) realizado com 402 pacientes submetidos a cirurgia colorretal, demonstrou que quase três quartos das pessoas desse estudo receberam alimentos sólidos no primeiro dia de pós-operatório, entretanto, apenas um terço tolerou alimentos sólidos precocemente, embora tenha sido utilizada uma definição não padronizada de tolerância. Outro realizado por Toledano S, et al. (2019) analisou a tolerância a dieta oral através da presença de retorno alimentar por via oral ou necessidade de uso de sonda nasogástrica com o avançar da dieta. Os resultados evidenciaram não haver diferença nessas variáveis entre o grupo de dieta pós operatória precoce e o grupo de dieta tardia.

Para Jochum SB, et al. (2020) e Aguilar-Nascimento JE, et al. (2009) a alimentação precoce após cirurgia colorretal é segura, pois permite menor tempo de permanência hospitalar sem aumentar os riscos de eventos adversos graves, como reoperação em 30 dias, embolia pulmonar, insuficiência renal aguda, acidente vascular encefálico, infarto agudo do miocárdio, sepse ou morte. Corroborando esses benefícios, Toledano $S$, et al. (2019) evidenciaram que a dieta precoce leva a um retorno acelerado da função intestinal e reduz o período de internação hospitalar em 2 a 4 dias, em comparação com grupo de alimentação tradicional. Esses desfechos foram observados em pacientes submetidos a cirurgias colorretais que necessitaram da realização de ileostomia ou colostomia.

Uma grande revisão de literatura realizada por Andersen HK, et al. (2006), utilizou 14 ensaios clínicos randomizados para evidenciar os benefícios da alimentação precoce nas cirurgias colorretais. Não se evidenciou nenhuma vantagem óbvia em manter os pacientes sem dieta após a cirurgia gastrointestinal, apoiando assim a antecipação da introdução da dieta, principalmente devido ao avanço nas técnicas cirúrgicas cada vez menos invasivas, que causam menos trauma cirúrgico.

Com o avançar dos estudos a aplicabilidade do Protocolo Acerto e ERAS passou a englobar procedimentos cirúrgicos que não somente os colorretais, entre eles as cirurgias do estômago. Um estudo realizado por Nakagawa M, et al. (2020), observou 91 pacientes com câncer gástrico submetidos a gastrectomia proximal ou total, e evidenciou que a ingestão alimentar oral precoce seja possível e segura para pacientes de baixo a moderado risco cirúrgico. Entretanto, deve ser aplicada com cautela para pacientes de alto risco, como portadores de hipertensão arterial sistêmica e diabetes mellitus, visto que esse grupo apresentou maior risco de vazamento através da anastomose esofagojejunal. Outro realizado por Chen J, et al. (2019), acompanhou 87 pacientes com câncer gástrico submetidos a gastrectomia parcial ou total, por via aberta ou laparoscópica e concluiu que a alimentação precoce pode encurtar o tempo de internação após a cirurgia sem aumentar a incidência de complicações.

Em comparação com o câncer colorretal a segurança e confiabilidade da alimentação oral precoce após cirurgia de câncer gástrico carecia de alto nível terapêutico de evidências. Este método não era adotado em cirurgia gástrica há muito tempo por medo de que a ingestão de alimentos acarretasse em vazamento anastomótico devido ao aumento da pressão intraluminal. Entretanto, nas últimas décadas diversos estudos mostraram que a alimentação oral precoce após gastrectomia é segura e viável (CHEN J, et al., 2019).

Outro ponto positivo a favor da aplicação da alimentação precoce nas cirurgias gástricas está relacionada ao estimulo hormonal excitatório após o procedimento cirúrgico. Reforçando tal fato, o estudo de Gao L, et al. (2019) sugere que a alimentação oral precoce regula a função fisiológica do trato intestinal e acelera a recuperação da função intestinal, estimulando a secreção excitatória de hormônios gastrointestinais, uma vez que o grupo que recebeu alimentação precocemente apresentou maiores níveis hormonais de gastrina e motilina. Sendo assim, a alimentação precoce em gastrectomias proximais e totais é segura e viável, com recuperação pós operatória mais rápida e sem aumentar o risco de efeitos adversos e complicações póscirúrgicas (WANG J, et al., 2019; GAO L, et al.,2019; NAKAGAWA M, et al.,2020).

No que diz respeito a cirurgias esofágicas os estudos buscam elucidar a diferença entre dieta enteral e dieta parenteral precoce. Para Mashhadi MTR, et al. (2015) os pacientes com diagnóstico de câncer esofágico também se beneficiam da realimentação precoce, uma vez que a alimentação enteral pode ser efetivamente utilizada nos primeiros dias após a esofagectomia, com poucas complicações precoces e resultados nutricionais semelhantes em comparação ao método parenteral. Além disso, a alimentação enteral precoce pode melhorar a resposta imune e reduzir custos hospitalares. 
A revisão sistemática e metanálise de Willcutts KF, et al. (2016) englobando 15 estudos e totalizando 2112 pacientes submetidos a cirurgias digestivas altas, incluindo gastrectomias, esofagectomias e procedimentos hepatobiliares, demonstrou que a alimentação oral pós-operatória precoce, em comparação com o manejo tradicional, está associada a um menor tempo de internação hospitalar e não está relacionada a um aumento das complicações pós-cirúrgicas, reforçando sua aplicabilidade e segurança.

Com relação à alimentação precoce de cirurgias abdominais de emergência, os estudos ainda são escassos se comparados às cirurgias eletivas. O estudo realizado por Klappenbach RF, et al. (2013), acompanhou 295 pacientes submetidos a cirurgias abdominais de emergência, revelou que a introdução da dieta desde o primeiro dia pós-cirúrgico foi associada à aumento de vômitos, porém tratados facilmente, além de diminuição da fome em comparação com pacientes alimentados tardiamente. Entretanto, outros benefícios relacionados à alimentação precoce, como morbidade reduzida, menor frequência de íleo pós-operatório e menor tempo de internação não foram demonstrados neste ensaio.

Já o estudo executado por Moydien MR, et al. (2016), acompanhou 78 pacientes vítimas de trauma abdominal penetrante que necessitaram de laparotomia de emergência, evidenciando que 12 pacientes alimentados precocemente apresentaram complicação após a cirurgia e o grupo de alimentação tardia apresentou 11 complicações, com isso não houve diferença estatisticamente significativa entre os dois grupos, porém o grupo de alimentação precoce teve menor tempo de internação hospitalar, por isso esse estudo acredita ser viável e seguro abreviação do jejum em cirurgias abdominais de emergência.

Por fim, para Le Guen M, et al. (2014) há divergências entre os estudos publicados com relação à melhora do íleo pós-operatório e tempo de permanência hospitalar nos grupo de alimentação pós-operatória tradicional e grupo de alimentação precoce, mas estes afirmam que os pacientes submetidos a cirurgia de emergência, exceto quando houver vômito pós-operatório imediato, provavelmente se beneficiam da mesma estratégia de alimentação oral precoce.

\section{CONSIDERAÇÕES FINAIS}

As práticas da medicina tradicional no que diz respeito à alimentação no pós-operatório estão ultrapassadas, e o surgimento de protocolos como ACERTO e ERAS proporcionam um cuidado padronizado baseado em evidências científicas que é benéfico para pacientes e equipe multidisciplinar. Apesar disso, é perceptível a dificuldade das equipes hospitalares em seguirem os novos protocolos e com isso não há padronização no manejo da alimentação, prejudicando a recuperação dos pacientes submetidos a cirurgias digestivas. Já é de conhecimento do meio científico as alterações metabólicas, inflamatórias e endócrinas acarretadas pelo trauma cirúrgico e como a nutrição pós-operatória adequada impacta positivamente na evolução clínica dos pacientes. Além disso, esse estudo confirma a segurança da alimentação precoce em cirurgias colorretais e digestivas altas com redução da morbimortalidade hospitalar, frequência de deiscência anastomótica, tempo de permanência hospitalar e intensificação da recuperação da função intestinal, consequentemente acelerando a recuperação após a cirurgia, bem como reduzindo os custos hospitalares durante a internação do paciente. Com relação a cirurgias abdominais de emergência, as pesquisas apesar de promissoras possuem divergências quanto a eficácia da alimentação precoce no pós-operatório, por isso ainda são necessários mais estudos clínicos randomizados para confirmar essas descobertas.

\section{REFERÊNCIAS}

1. AGUILAR-NASCIMENTO JE, et al. Abordagem multimodal em cirurgia colorretal sem preparo mecânico de cólon. Revista do Colégio Brasileiro de Cirurgiões, v. 36, n. 3, p. 204-209, 2009.

2. AGUILAR-NASCIMENTO JE, et al. Acerto pós-operatório: avaliação dos resultados da implantação de um protocolo multidisciplinar de cuidados peri-operatórios em cirurgia geral. Revista do Colégio Brasileiro de Cirurgiões, v. 33, n. 3, p. 181-188, 2006.

3. AGUILAR-NASCIMENTO JE, et al. Diretriz ACERTO de intervenções nutricionais no perioperatório em cirurgia geral eletiva. Revista do Colégio Brasileiro de Cirurgiões, v. 44, n. 6, p. 633-648, 2017.

4. AGUILAR-NASCIMENTO JE, et al. Ingestão pré-operatória de carboidratos diminui a ocorrência de sintomas gastrointestinais pós-operatórios em pacientes submetidos à colecistectomia. ABCD. Arquivos Brasileiros de Cirurgia Digestiva (São Paulo), v. 20, n. 2, p. 77-80, 2007 
5. AKBARSHAHI H, et al. Perioperative nutrition in elective gastrointestinal surgery-potential for improvement? Digestive surgery, v. 25, n. 3, p. 165-174, 2008.

6. ANDERSEN HK, et al. Early enteral nutrition within $24 \mathrm{~h}$ of colorectal surgery versus later commencement of feeding for postoperative complications. Cochrane Database of Systematic Reviews, n. 4, 2006.

7. ATKINSON C, et al. Factors associated with early postoperative feeding: An observational study in a colorectal surgery population. Clinical Nutrition ESPEN, 2020.

8. BINDEROW SR, et al. Must early postoperative oral intake be limited to laparoscopy? Diseases of the colon \& rectum, v. 37, n. 6, p. 584-589, 1994.

9. BUFO AJ, et al. Early postoperative feeding. Diseases of the colon \& rectum, v. 37, n. 12, p. 1260-1265, 1994.

10. CHEN J, et al. Effects of a stepwise, local patient-specific early oral feeding schedule after gastric cancer surgery: a single-center retrospective study from China. Scientific reports, v. 9, n. 1, p. 1-9, 2019.

11. CORREIA MITD, SILVA RG. Paradigmas e evidências da nutrição peri-operatória. Revista do Colégio Brasileiro de cirurgiões, v. 32, n. 6, p. 342-347, 2005.

12. COSTA HCBAL, et al. Resultados clínicos antes e após a implantação do protocolo ACERTO. Revista do Colégio Brasileiro de Cirurgiões, v. 40, n. 3, p. 174-179, 2013.

13. DE LUIS DA, et al. Surgical infection and malnutrition. Nutricion hospitalaria, v. 30, n. 3, p. 509-513, 2014.

14. DERVENIS $\mathrm{C}$, et al. Benefits and limitations of enteral nutrition in the early postoperative period. Langenbeck's archives of surgery, v. 387, n. 11-12, p. 441-449, 2003.

15. DIFRONZO LA, et al. Benefits of early feeding and early hospital discharge in elderly patients undergoing open colon resection. Journal of the American College of Surgeons, v. 197, n. 5, p. 747-752, 2003.

16. DIFRONZO LA, et al. Factors affecting early postoperative feeding following elective open colon resection. Archives of Surgery, v. 134, n. 9, p. 941-946, 1999.

17. FRANCISCO SC, et al. Jejum em pacientes cirúrgicos eletivos: comparação entre o tempo prescrito, praticado e o indicado em protocolos de cuidados perioperatórios. ABCD. Arquivos Brasileiros de Cirurgia Digestiva (São Paulo), v. 28, n. 4, p. 250-254, 2015.

18. GAO L, et al. Effect of early oral feeding on gastrointestinal function recovery in postoperative gastric cancer patients: a prospective study. J BUON, v. 24, p. 194-200, 2019.

19. GIANOTTI L, et al. Safety, feasibility, and tolerance of early oral feeding after colorectal resection outside an enhanced recovery after surgery (ERAS) program. International journal of colorectal disease, v. 26, n. 6, p. 747-753, 2011.

20. HARTSELL $P$, et al. Early postoperative feeding after elective colorectal surgery. Archives of Surgery, v. 132, n. 5, p. 518-521, 1997.

21. JOCHUM SB, et al. Early feeding in colorectal surgery patients: safe and cost effective. International Journal of Colorectal Disease, v. 35, n. 3, p. 465-469, 2020.

22. KLAPPENBACH RF, et al. Early oral feeding versus traditional postoperative care after abdominal emergency surgery: a randomized controlled trial. World journal of surgery, v. 37, n. 10, p. 2293-2299, 2013.

23. LE GUEN M, et al. Early oral feeding after emergency abdominal operations: another paradigm to be broken? Current Opinion in Clinical Nutrition \& Metabolic Care, v. 17, n. 5, p. 477-482, 2014.

24. LUDWIG RB, et al. Menor tempo de jejum pré-operatório e alimentação precoce no pós-operatório são seguros? ABCD. Arquivos Brasileiros de Cirurgia Digestiva (São Paulo), v. 26, n. 1, p. 54-58, 2013.

25. MASHHADI MTR, et al. Early post operative enteral versus parenteral feeding after esophageal cancer surgery. Iranian journal of otorhinolaryngology, v. 27, n. 82, p. 331, 2015.

26. MOYDIEN MR, et al. Enhanced recovery after surgery (ERAS) in penetrating abdominal trauma: A prospective singlecenter pilot study. South African Journal of Surgery, v. 54, n. 4, p. 7-10, 2016.

27. NAKAGAWA M, et al. Feasibility and safety of early oral intake and discharge after total or proximal gastrectomy: an analysis of consecutive cases without exclusion criteria. Annals of Surgical Oncology, v. 27, n. 3, p. 812-821, 2020.

28. ORTIZ H, et al. Is early postoperative feeding feasible in elective colon and rectal surgery? International journal of colorectal disease, v. 11, n. 3, p. 119-121, 1996.

29. OSLAND E, et al. Early versus traditional postoperative feeding in patients undergoing resectional gastrointestinal surgery: a meta-analysis. Journal of parenteral and enteral nutrition, v. 35, n. 4, p. 473-487, 2011.

30. PETRELLI NJ, et al. Early postoperative oral feeding after colectomy: an analysis of factors that may predict failure. Nutrition in Clinical Practice, v. 17, n. 5, p. 323-324, 2002.

31. RATTRAY M, et al. A systematic review of feeding practices among postoperative patients: is practice in-line with evidenced-based guidelines? Journal of Human Nutrition and Dietetics, v. 31, n. 2, p. 151-167, 2018.

32. RATTRAY M, et al. Hospital Staffs' Perceptions of Postoperative Nutrition Among Colorectal Patients: A Qualitative Study. Nutrition in Clinical Practice, v. 35, n. 2, p. 306-314, 2020.

33. SÁNCHEZ A PAPAPIETRO K. Nutrición perioperatoria en protocolos quirúrgicos para una mejor recuperación postoperatoria (Protocolo ERAS). Revista médica de Chile, v. 145, n. 11, p. 1447-1453, 2017.

34. SILVA VB, et al. Tempo de jejum em perioperatório de cirurgias gastrintestinais. Rev Bras Nutr Clin, v. 30, n. 2, p. 136-40, 2015.

35. STOCCHE RM, et al. Anestesia e resposta neuroendócrina e humoral ao estresse cirúrgico. Rev. bras. anestesiologia, p. 59-69, 2001.

36. TOLEDANO S, et al. Exploring the Differences Between Early and Traditional Diet Advancement in Postoperative Feeding Outcomes in Patients with an lleostomy or Colostomy. Nutrition in Clinical Practice, v. 34, n. 4, p. 631-638, 2019.

37. WANG J, et al. Comparison of early oral feeding with traditional oral feeding after total gastrectomy for gastric cancer: A propensity score matching analysis. Frontiers in Oncology, v. 9, p. 1194, 2019.

38. WEIMANN A, et al. ESPEN guideline: clinical nutrition in surgery. Clinical nutrition, v. 36, n. 3, p. 623-650, 2017.

39. WILLCUTTS KF, et al. Early oral feeding as compared with traditional timing of oral feeding after upper gastrointestinal surgery. Annals of surgery, v. 264, n. 1, p. 54-63, 2016. 